\title{
The Predictive Value of Neutrophil-to- Lymphocyte Ratio and Platelet-to-Lymphocyte Ratio Levels of Diabetic Peripheral Neuropathy
}

\author{
Meiqiao Chen ${ }^{1,2, *}$ \\ Yuyou Zhu ${ }^{2, *}$ \\ Jumei Wang ${ }^{3}$ \\ Guoping Wang ${ }^{2}$ \\ Yuanbo $\mathrm{Wu}^{2}$
}

'Department of Geriatrics, The First Affiliated Hospital of USTC, Division of Life Sciences and Medicine, University of Science and Technology of China, Hefei, Anhui, 23000I, People's Republic of China; ${ }^{2}$ Department of Neurology, The First Affiliated Hospital of USTC, Division of Life Sciences and Medicine, University of Science and Technology of China, Hefei, Anhui Province, 23000I, People's Republic of China; ${ }^{3}$ Department of Endocrinology, The First Affiliated Hospital of USTC, Division of Life Sciences and Medicine, University of Science and Technology of China, Hefei, Anhui Province, 23000I, People's

Republic of China

*These authors contributed equally to this work
Correspondence: Yuanbo Wu Department of Neurology, The First Affiliated Hospital of USTC, Division of Life Sciences and Medicine, University of Science and Technology of China, Hefei, Anhui Province, 23000I, People's Republic of China

Email wuyuanbo@mail.ustc.edu.cn
Objective: This study was designed to assess the levels of neutrophil-to-lymphocyte ratio (NLR) and platelet-to-lymphocyte ratio (PLR) in diabetes patients to determine their prognostic value in predicting the disease of diabetic peripheral neuropathy (DPN).

Methods: We recruited 225 diabetes cases from the department of endocrinology of Anhui Provincial Hospital from August 2018 to October 2019. A total of 103 patients without diabetic peripheral neuropathy (DPN) were followed up for 18 months, and the number of patients of newly diagnosed DPN was counted. According to the results of neuroelectrophysiological examination, these patients were divided into the diabetes mellitus (DM) without DPN group and the DM with DPN group. The general information and results of blood samples were collected. The collected data were compared between groups, and the receiver operating characteristic curve (ROC) was drawn. The follow-up data were compared between groups and Binary Logistic regression analysis was performed.

Results: Patients with DPN shared distinct characteristics. For example, the patients were older, and had higher levels of inflammatory indicators (ie, levels of PLR and NLR), and lower level of indirect bilirubin, compared with patients without DPN. According to the receiver operating characteristic curve analysis, for type 1 diabetes, PLR showed the highest area under the curve (0.753). For type 2 diabetes, NLR showed the highest AUC of 0.602 . For the follow-up results, patients with newly diagnosed DPN bad higher NLR level.

Conclusion: If patients of type 1 and type 2 diabetes are combined with elevated level of PLR and NLR, respectively, they are more likely complicated with DPN. NLR and PLR could be used as predictors to help clinicians screening for DPN in different types of diabetes. For type 1 diabetes, if patients who were without DPN had higher NLR level, the risk of developing DPN in the future will be greatly increased.

Keywords: diabetes mellitus, diabetic peripheral neuropathy, neutrophil-to-lymphocyte ratio, platelet-to-lymphocyte ratio

\section{Introduction}

As defective insulin secretion or impaired biological function, chronic hyperglycemia can cause damage to various tissues and systems, especially eyes, kidneys, blood vessels and nerves. ${ }^{1}$ Most diabetes patients can be divided into two types. Type 1 diabetes mellitus (T1DM), due to the absolute lack of insulin secretion, can usually be identified by serological evidence and genetic markers of islet autoimmunity. Abnormal inflammation and immune responses are associated with the development of T1DM. Recent study have shown that innate immunity and 
inflammatory mediators play an important and wideranging roles, possibly inhibiting $\beta$-cell function, ${ }^{2}$ promoting subsequent apoptotic processes, and leading to insulin resistance in surrounding tissues.

Type 2 diabetes mellitus (T2DM), the more common type, accounts for $90 \sim 95 \%$ of diabetes, due to the insulin resistance (IR) and inadequate compensatory secretory response. Factors that contribute to impaired glucose tolerance (IGT) and IR include genetic factors, environmental factors, age, obesity, and inflammation. Activation of adipose tissue may lead to the release of inflammatory cytokines associated with IR, such as TNF- $\alpha$, leptin, IL6, resistin, monocyte chemotactic protein-1 (MCP-1), angiotensin, endolipids, retinol-binding protein-4, and serum amyloid A (SAA). ${ }^{1,2}$ Inflammatory factors induce and maintain the inflammatory response and inflammatory damage during the development of diabetes.

As one of the complications of diabetes mellitus (DM), diabetic peripheral neuropathy (DPN) usually develops insidiously and gradually. It can manifest as pain, numbness, tingling, weakness, and balance disorders, leading to ulcers, gangrene, and even amputation. Some patients are asymptomatic at an early stage, which may lead to neglect of the disease. Therefore, early detection and treatment of DPN play an important role in improving disease prognosis and life quality. ${ }^{3}$ The development of DPN is related to metabolic disorders, such as oxidative stress, increased polyol flux, accumulation of glycosylated end products and lipid changes, and other metabolic abnormalities. ${ }^{4}$ To date, some hemogram derived inflammatory markers and related metabolites have been found to be associated with diabetes mellitus. Mean platelet volume (MPV) can provide important information on the course and prognosis in many inflammatory conditions. ${ }^{5}$ Red blood cell distribution width (RDW) is associated with cardiovascular disease, sepsis, and tumors. ${ }^{6-8}$ Besides, recent studies have shown that NLR and RLR can be used as systemic marker in some inflammatory conditions including cardiovascular disease, metabolic syndrome and malignancies. ${ }^{9-11}$ Both of them are novel, available, and inexpensive marker of Inflammatory status. Against this background, we aimed to study the association between the occurrence of DPN and related indicators in patients with type 1 or type 2 diabetes, and patients without DPN were followed up to investigate the predictive value of these indicators on newly diagnosed DPN.

\section{Materials and Methods}

\section{Subjects}

In this study, 225 consecutively hospitalized patients were recruited from August 31, 2018 to October 1, 2019 at the First Affiliated Hospital of University of Science and Technology of China. The inclusion criteria included a diagnosis of diabetes with or without the symptoms and signs of DPN. Diabetes was diagnosed using the revised American diabetes association standards, including fasting plasma glucose $[\mathrm{FPG}] \geq 7.0 \mathrm{mmol} / \mathrm{L}[126 \mathrm{mg} / \mathrm{dL}]$ and/or postprandial $2 \mathrm{~h}$ glucose value $\geq 11.1 \mathrm{mmol} / \mathrm{L}[200 \mathrm{mg} / \mathrm{dL}]{ }^{12}$ Exclusion criteria were as follows: a) a history of multiple nerves due to other causes, such as hereditary, alcoholic, metabolic, inflammatory, and toxic factors; b) a history of tumor radiotherapy and chemotherapy; c) skin damage or swelling which can interfere with nerve conduction; d) active infection and using of medicine affecting the white blood cell counts; e) Complicated hematogenous disease or rheumatic disease; f) a prior history of leg or ankle fractures or surgery. Patients without DPN were followed up in the following 18 months, and patients with new-developed DPN were counted. This study gained approval by the Chinese Clinical Trial Registry's ethics committee, and informed consent was obtained from all enrolled patients. This study's clinical registration number is ChiCTR1900026629. This study was conducted in accordance with the Declaration of Helsinki. Due to the limited number of ethical review staff in our hospital, we had to queue for a long time, so we chose to get approval from the Ethics Committee of the Chinese Clinical Trial Registry.

\section{Data Collection}

We collected data on patient characteristics (eg, age, type of diabetes, disease course, medical history, height, weight) and inflammatory indicators (eg, levels of NLR and PLR) using the hospital's electronic medical record system. The included subjects were divided into four different groups, including T1DM with DPN group (T1DPN group), T1DM without DPN group (T1DM group), T2DM with DPN group (T2DPN group), T2DM without DPN group (T2DM group). The diagnostic criteria for DPN were based on the Toronto Expert Consensus. ${ }^{13}$ Professional doctors (Meichao Chen and Yuanbo $\mathrm{Wu}$ ) verified the data.

\section{Statistical Analysis}

Statistical software SPSS, version 20.0, was used to analyze the collected data. Continuous data and normally 
distributed data were expressed as the mean \pm standard deviation using the Student's $t$-test for intergroup comparisons, whereas non-normally distributed data were expressed as the median $(1 / 4,3 / 4)$ using the MannWhitney $U$-test. Categorical variables were expressed as counts (\%) using the $\chi^{2}$ test for comparisons. The influence of related indicators levels were assessed using binary logistic regression analysis with significant factors. Results were expressed as adjusted odds ratios (OR) with the corresponding 95\% confidence intervals (CI). Receiver operating characteristic (ROC) curves were drawn, cut-off values were determined. The cut-off values and their corresponding sensitivity and specificity were determined using the Youden index. Drew the corresponding box diagram for the indicator with the maximum AUC. $P$ values less than 0.05 were considered statistically significant.

\section{Results}

\section{General Data}

A total of 70 patients with type 1 diabetes were recruited, including 48 patients with DPN and 22 patients without DPN. For type 2 diabetes, 155 patients were recruited, including 74 patients with DPN and 81 patients without DPN.

Data were compared between the two groups in terms of general patient characteristics (eg, age, disease course), inflammatory indicators (eg, levels of NLR and PLR).

For type 1 diabetes, age was statistically different between the two groups. The age of patients with DPN was generally higher than that of patients without DPN $(36.31 \pm 15.64$ years and $28.32 \pm 12.79$ years, respectively). For type 2 diabetes, age, disease course and systolic blood pressure were statistically different between the two groups. The age of patients with DPN was generally higher than that of patients without DPN (61.92 \pm 11.22 years and $55.90 \pm 11.34$ years, respectively). The disease course of patients with DPN was longer than that of patients without DPN (11.00 (5.00, 19.50) years and 6.00 $(2.00,10.00)$ years, respectively). The systolic blood pressure of patients with DPN was higher than that of patients without DPN (144.07 \pm 20.60 years and 136.47 \pm 17.12 $\mathrm{mmHg}$, respectively). The baseline characteristics of hospitalized patients are shown in Table 1 .

For type 1 diabetes, levels of platelet counts, indirect bilirubin, total cholesterol, NLR and PLR were all statistically different between the two patient groups, and for type 2 diabetes, indirect bilirubin, triglyceride and NLR were all statistically different between the two patient groups. Notably, the inflammatory indices of patients with DPN were generally higher than those of patients without DPN, as shown in Table 1.

\section{Predictive Value of NLR, PLR and I-BIL}

Between DPN group and DM group, ROC curves were drawn for NLR, PLR and I-BIL. The AUCs and cut-off values were calculated according to their specificity and sensitivity as predictive factors.

The AUC of PLR levels was 0.753 (95\% CI 0.6350.871 ); the sensitivity was $70.80 \%$ and the specificity was $77.30 \%$ for predicting DPN in type 1 diabetes when the cut-off level of PLR was 97.880. The cut-off NLR level was set at 2.485, with a sensitivity of $38.00 \%$ and a specificity of $79.00 \%$, for predicting disease severity, and an AUC of 0.602 (95\% CI 0.513 0.691), as shown in Figure 1 and Table 2.

\section{Comparison of NLR and PLR in Different Groups}

The NLR level in T2DM with DPN group was statistically higher than that of T2DM without DPN group, T1DM with DPN group and T1DM without DPN group. While the PLR level in the T1DM with DPN group was significantly higher than that of T1DM without DPN group, T2DM with DPN group and T2DM without DPN group, as shown in Figures 2 and 3.

\section{Follow-Up Results General Data}

After 18 months of follow-up for diabetes patients without DPN, 9 patients of type 1 diabetes were newly diagnosed with DPN, while 14 patients of type 2 diabetes were newly diagnosed with DPN. We analyzed the data between patients with and without newly diagnosed DPN. The type of diabetes mellitus was a significant factor for the new onset of DPN, and the BWI of patients without DPN was higher than that of patients with newly diagnosed DPN. The results are shown in Table 3.

For the relative indicator, levels of NLR, PLR, NEUT (\%), NC (109/L), LYMPH (\%), LC (109/L), TC (mmol/L), TG $(\mathrm{mmol} / \mathrm{L})$ and LDL-C $(\mathrm{mmol} / \mathrm{L})$ were all statistically different between the two patient groups. For the inflammatory indicators, levels of PLR and NLR were all statistically higher in the group with newly diagnosed DPN. For lipid 


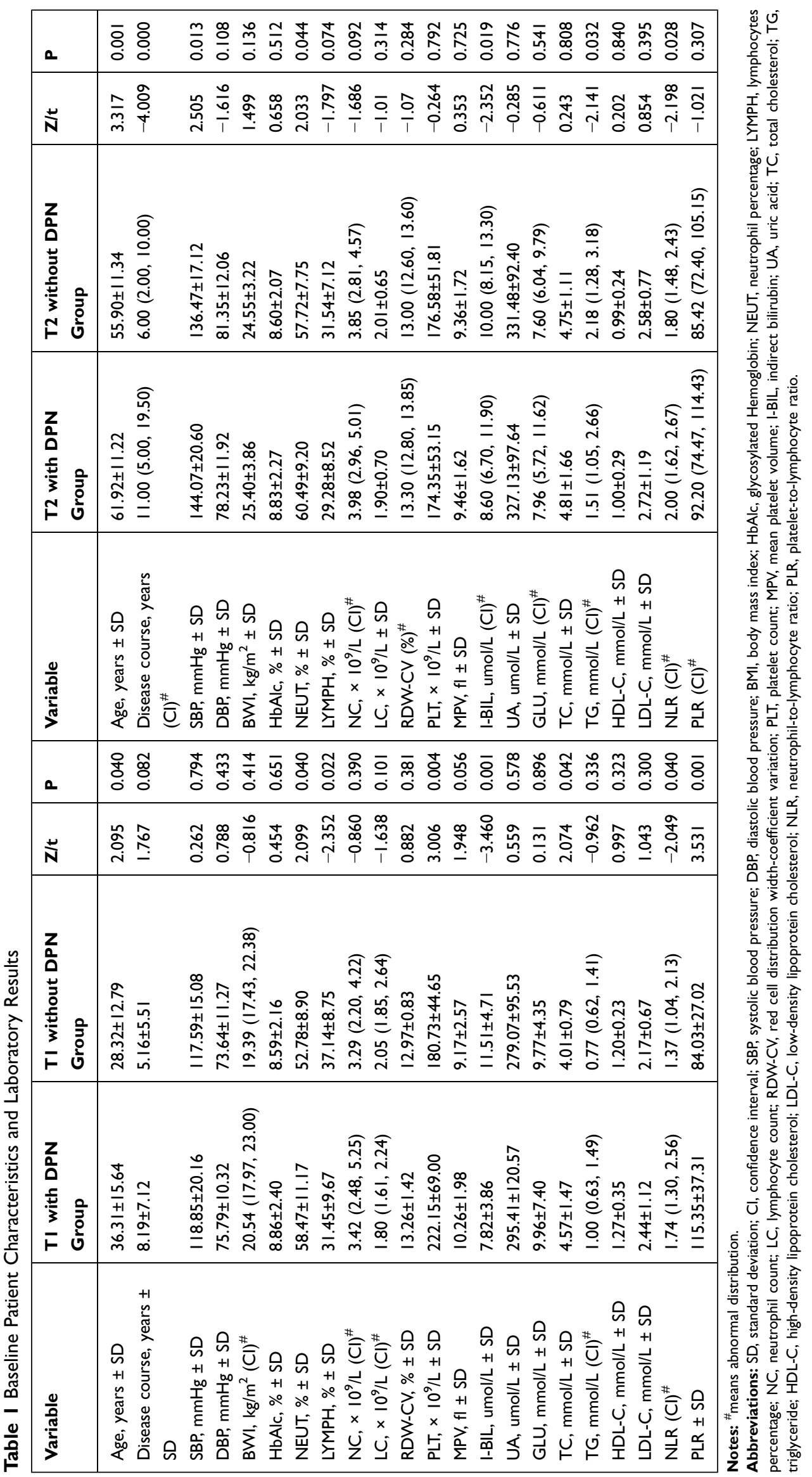



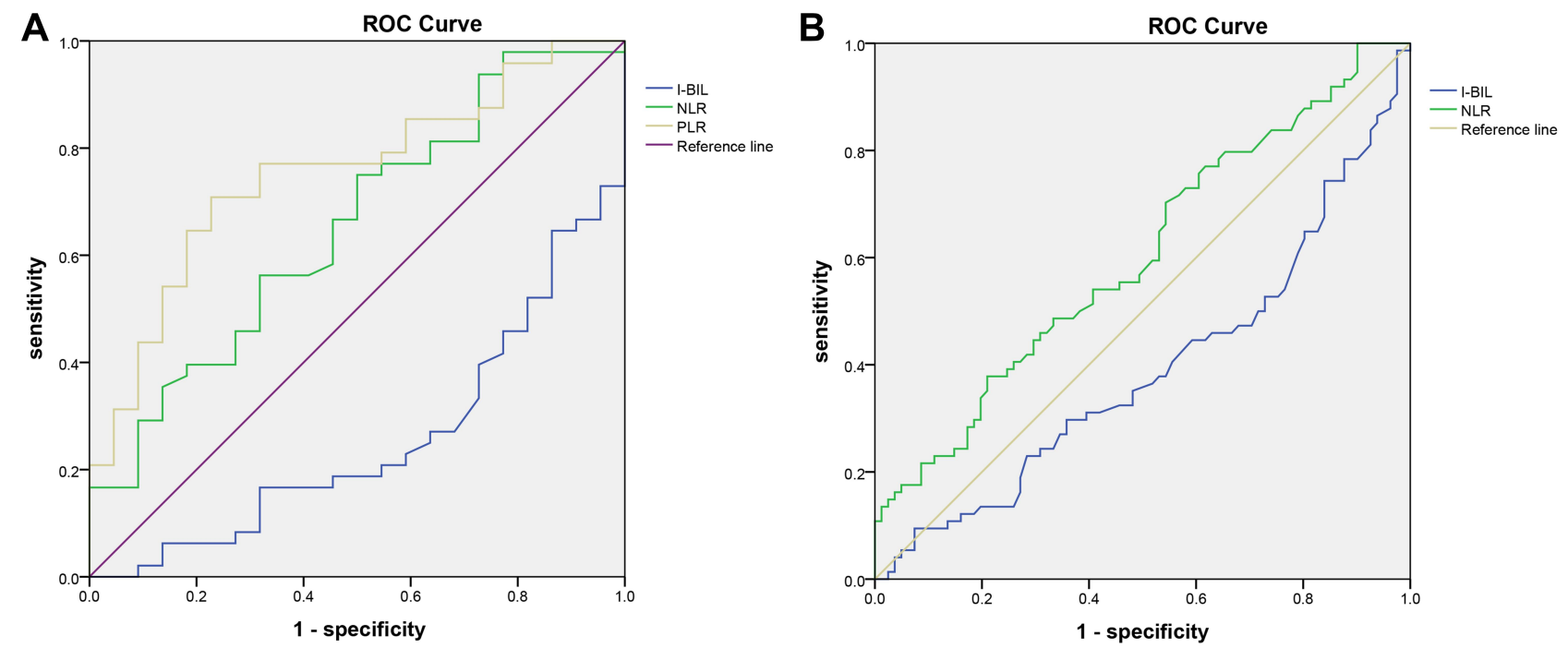

Figure I The ROC curves of predicting whether diabetic patients combined with DPN. Subfigure (A) is the receiver operating characteristic analysis (ROC) for NLR, PLR and I-BIL to predict DPN in TIDM. Subfigure (B) is the ROC curve of NLR and I-BIL for predicting whether T2DM is combined with DPN.

metabolism-related indexes, TC $(\mathrm{mmol} / \mathrm{L}), \mathrm{TG}(\mathrm{mmol} / \mathrm{L})$ and LDL-C (mmol/L) were significantly lower in the group with newly diagnosed DPN. The results are shown in Table 3.

\section{Regression Model}

After adjusting for above recorded confounders such as BWI, TC, TG, LDL-C, type of diabetes and NLR were associated with the new diagnosis of DPN in multivariate binary logistic regression analysis. The adjusted OR were 0.091 (95\% CI, 0.010-0.799) and 0.060 (95\% CI, 0.014 $0.258 ; \mathrm{p} \leq 0.001)$, respectively. The results are shown in Table 4 .

\section{Discussion}

The neuropathy of diabetes is the most common neurological disorder in the world, and its prevalence increases with the extension of diabetes. ${ }^{14}$ It affects about half of people with diabetes, affecting their sensorimotor function.
And the early stages of DPN can be asymptomatic, resulting in delaying diagnosis. Seeking an effective and convenient screening method can improve the screening efficiency.

Multiple factors contribute to the occurrence of DPN, including endothelial injury, microvascular dysfunction, metabolic disorders, oxidative stress, abnormal cytokines and immune factors, among which inflammatory injury plays an important role. Chronic hyperglycemia can lead to microcirculation disorders. A series of vascular pathological changes can occur, such as vascular endothelial cell proliferation, microvascular basement membrane thickening and hyaline degeneration, which leads to direct narrowing of lumen. The increase of blood viscosity and the disturbance of blood flow aggravate the reduction of blood supply to local tissues. This process leads to ischemia and hypoxia of nerve tissues, stimulating the increase of cytokines, and aggravating inflammatory damage. Besides, hyperglycemia

Table 2 ROC Curve Area and Cut-Off Values of NLP PLR and I-Bil for the Diagnosis of DPN

\begin{tabular}{|l|l|l|l|l|l|}
\hline \multicolumn{2}{|l|}{} & \multicolumn{2}{l|}{ TIDM } & T2DM & \multicolumn{2}{l|}{} \\
\cline { 2 - 6 } & I-Bil & NLR & PLR & I-Bil & NLR \\
\hline AUC $(95 \% \mathrm{Cl})$ & $0.745(0.625 \sim 0864)$ & $0.652(0.515 \sim 0.79)$ & $0.753(0.635 \sim 0.87 I)$ & $0.390(0.301 \sim 0.480)$ & $0.602(0.513 \sim 0.691)$ \\
P value & 0.001 & 0.042 & 0.001 & 0.019 & 0.028 \\
Cut-off value & 8.650 & 1.325 & 97.880 & 16.950 & 2.485 \\
Sensitivity & 0.682 & 0.750 & 0.708 & 0.095 & 0.380 \\
Specificity & 0.729 & 0.500 & 0.773 & 0.926 & 0.790 \\
\hline
\end{tabular}

Abbreviations: I-BIL, indirect bilirubin; NLR, neutrophil-to-lymphocyte ratio; PLR, platelet-to-lymphocyte ratio. 

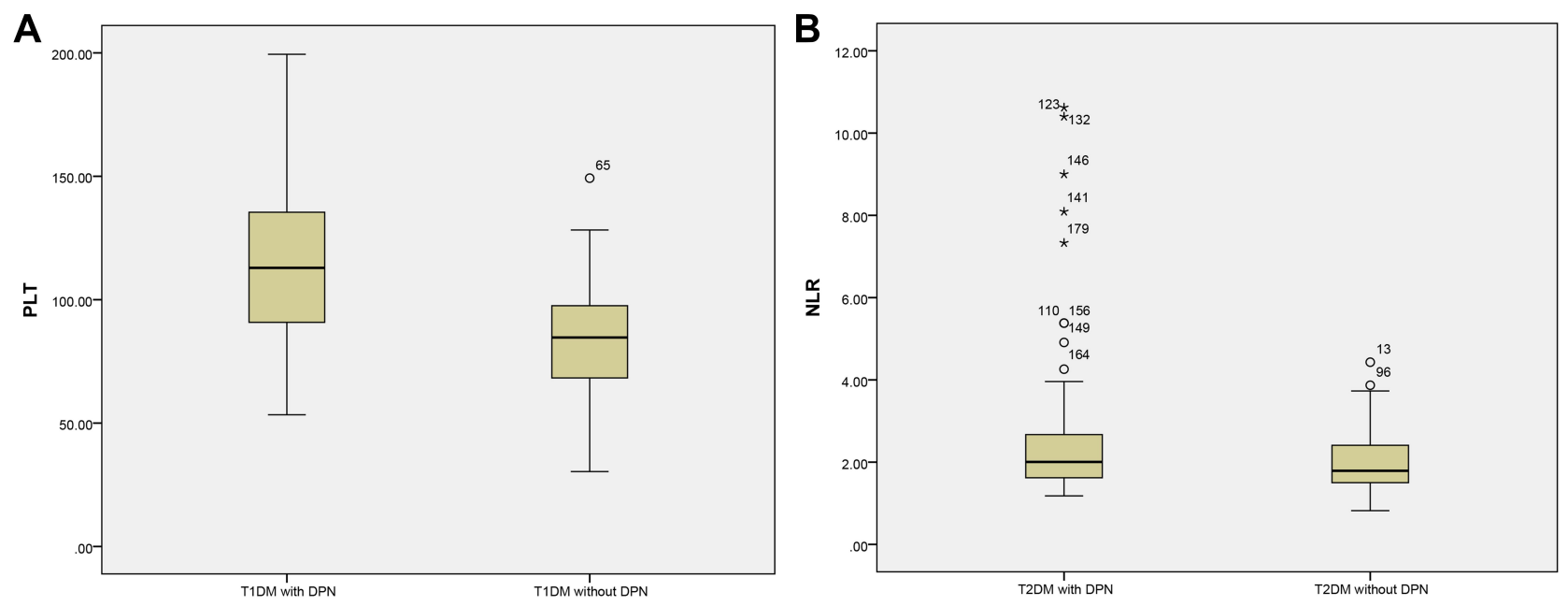

Figure 2 The comparison of the PLR and NLR values of DPN for 2 types of diabetes. Subfigure (A) is the comparison of the PLR value between the TIDM with DPN group and TIDM without DPN group. Subfigure $(\mathbf{B})$ is the comparison of the NLR value between the T2DM with DPN group and T2DM without DPN group.
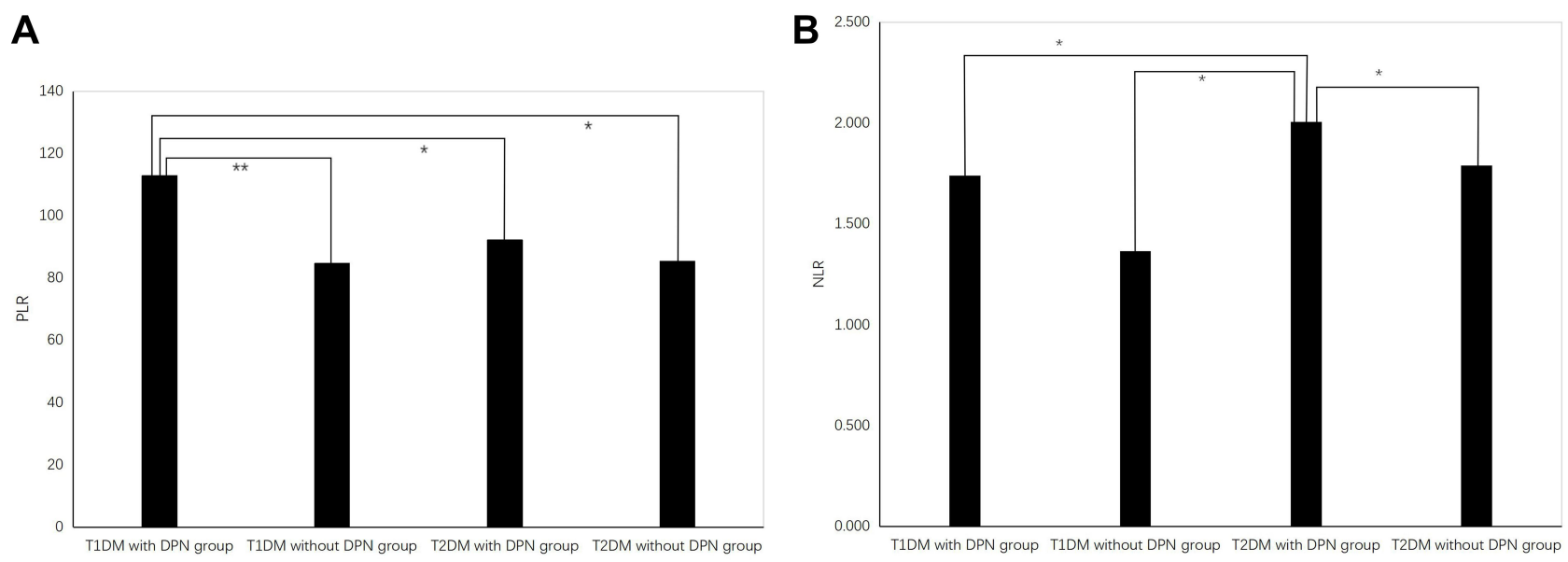

Figure 3 Bar chart of NLR and PLR values for each group. Subfigure $(\mathbf{A})$ is a bar diagram for four sets of PLR values. Subfigure $(\mathbf{B})$ reacts NLR values for four groups; $P<0.05$ was marked as “*”, and $P<0.01$ as “***”.

leads to damage through several major, wellcharacterized biochemical pathways, including activation of the polyol pathway, increased levels of advanced glycation end products (AGEs) and their receptors, activation of protein kinase $\mathrm{C}$ (PKC), ${ }^{15}$ mitogen-activated protein kinase (MAPK), and inducible nitric oxide synthase. ${ }^{16}$ These biochemical processes can produce oxidative mediators and inflammatory mediators, resulting in local or systemic tissue damage. Abnormal lipid metabolism is also one of the important influencing factors. Adipocytes are important components for inducing and maintaining the inflammatory response. In general, inflammation injury, activated and maintained by various pathways, plays an important role in the development of diabetes mellitus and its complications.
Neutrophil-to-lymphocyte ratio (NLR) represents the balance of neutrophils and lymphocytes in vivo. Neutrophils are closely related to inflammatory responses, and lymphocytes reflect immune regulatory pathways. ${ }^{17,18}$ They can reflect systemic inflammation, ${ }^{19,20}$ as well as innate immune responses (mediated by neutrophils) and adaptive immune responses (mediated by lymphocytes). ${ }^{21}$ The nonspecific inflammatory response caused by hyperglycemia may lead to changes in peripheral blood cell levels, which may explain the abnormal NLR values. Association between inflammatory conditions and elevated NLR has been well-established. ${ }^{22}$ The reason NLR is reported as a novel marker is that it is very stable compared with the absolute count, which can be altered by various physical, physiological and pathological factors. ${ }^{23}$ 
Table 3 Baseline Patient Characteristics and Laboratory Results on Follow-Up Subjects

\begin{tabular}{|c|c|c|c|c|c|}
\hline & & Newly Diagnosed DPN Group & DM without DPN Group & $\chi^{2} / \mathbf{Z} / \mathbf{t}$ & $\mathbf{P}$ \\
\hline \multirow[t]{2}{*}{ Type of diabetes, n (\%) } & Type I & $9(40.91 \%)$ & $13(59.09 \%)$ & 3.903 & 0.048 \\
\hline & Type 2 & 14 (20.0\%) & 56 (80.0\%) & & \\
\hline \multicolumn{2}{|l|}{ Age, years \pm SD } & $48.83 \pm 18.86$ & $49.2 \pm 15.99$ & -0.094 & 0.926 \\
\hline \multicolumn{2}{|c|}{ Disease course, years \pm SD } & $8.81 \pm 6.85$ & $6.02 \pm 5.75$ & 1.919 & 0.058 \\
\hline \multicolumn{2}{|l|}{$\mathrm{SBP}, \mathrm{mmHg} \pm \mathrm{SD}$} & $|3| .78 \pm 2 \mid .69$ & $|32.54 \pm| 7.57$ & -0.168 & 0.867 \\
\hline \multicolumn{2}{|l|}{$\mathrm{DBP}, \mathrm{mmHg} \pm \mathrm{SD}$} & $76.7 \pm 11.7$ & $80.74 \pm 12.63$ & -1.407 & 0.167 \\
\hline \multicolumn{2}{|l|}{ BWI, kg/m2 (Cl) } & $21.63(19.16,23.39)$ & $23.62(21.61,26.64)$ & -2.574 & 0.010 \\
\hline \multicolumn{2}{|l|}{ HbAlc, \% \pm SD } & $8.66 \pm 1.39$ & $8.64 \pm 2.28$ & 0.040 & 0.968 \\
\hline \multicolumn{2}{|l|}{ NEUT, \% (Cl) $)^{\#}$} & $65.4(57.1,70.4)$ & $54.4(50.55,59.75)$ & -4.153 & 0.000 \\
\hline \multicolumn{2}{|l|}{ LYMPHT, \% $(\mathrm{Cl})^{\#}$} & $23.4(20.7,30.7)$ & $34.7(29.65,38.25)$ & -4.157 & 0.000 \\
\hline \multicolumn{2}{|l|}{$\mathrm{NC}, \times 10^{9} / \mathrm{L} \pm \mathrm{SD}$} & $4.37 \pm 1.82$ & $3.59 \pm 1.21$ & 2.336 & 0.022 \\
\hline \multicolumn{2}{|l|}{$\mathrm{LC}, \times 10^{9} / \mathrm{L}(\mathrm{Cl})^{\#}$} & I.7I $(1.33,1.99)$ & $2.05(1.81,2.53)$ & -3.481 & 0.000 \\
\hline \multicolumn{2}{|l|}{ RDW-CV, \% \pm SD } & $13.57 \pm 1.38$ & $13.16 \pm 0.85$ & 1.321 & 0.197 \\
\hline \multicolumn{2}{|l|}{$\mathrm{PLT}, \times 10^{9} / \mathrm{L} \pm \mathrm{SD}$} & $|74.9| \pm 44.8 \mid$ & $182.17 \pm 52.25$ & -0.597 & 0.552 \\
\hline \multicolumn{2}{|l|}{$M P V, f l \pm S D$} & $9.04 \pm 2.41$ & $9.44 \pm 1.8$ & -0.853 & 0.396 \\
\hline \multicolumn{2}{|l|}{ I-BIL, umol/L $\pm S D$} & $9.61 \pm 4.22$ & $11.75 \pm 5.84$ & -1.615 & 0.110 \\
\hline \multicolumn{2}{|l|}{$\mathrm{UA}, \mathrm{umol} / \mathrm{L} \pm \mathrm{SD}$} & $302.03 \pm|| 4.0 \mid$ & $323.97 \pm 91.25$ & -0.936 & 0.352 \\
\hline \multicolumn{2}{|l|}{$\mathrm{GLU}, \mathrm{mmol} / \mathrm{L} \pm \mathrm{SD}$} & $9.24 \pm 5.9$ & $8.98 \pm 4.14$ & 0.227 & 0.821 \\
\hline \multicolumn{2}{|l|}{$\mathrm{TC}, \mathrm{mmol} / \mathrm{L} \pm \mathrm{SD}$} & $4.17 \pm 1.1$ & $4.7 I \pm 1.09$ & -2.039 & 0.044 \\
\hline \multicolumn{2}{|l|}{$\mathrm{TG}, \mathrm{mmol} / \mathrm{L}(\mathrm{Cl})^{\#}$} & $0.83(0.68,1.61)$ & $2.12(1.26,3.28)$ & -3.314 & 0.001 \\
\hline \multicolumn{2}{|l|}{ HDL-C, $\mathrm{mmol} / \mathrm{L} \pm \mathrm{SD}$} & $1.07 \pm 0.28$ & $1.02 \pm 0.25$ & 0.699 & 0.486 \\
\hline \multicolumn{2}{|l|}{ LDL-C, mmol/L (Cl) } & $2.04(1.78,2.6)$ & $2.59(2.01,3.07)$ & -2.620 & 0.009 \\
\hline \multicolumn{2}{|l|}{$\operatorname{NLR}(\mathrm{Cl})^{\#}$} & $2.88(1.82,3.35)$ & $1.57(1.32,2.01)$ & -4.215 & 0.000 \\
\hline \multicolumn{2}{|l|}{$\operatorname{PLR}(\mathrm{Cl})^{\#}$} & $96.46(86.87,124.79)$ & $83.63(70.48,93.47)$ & -3.197 & 0.001 \\
\hline
\end{tabular}

Note: ${ }^{\#}$ means abnormal distribution.

Abbreviations: SD, standard deviation; Cl, confidence interval; SBP, systolic blood pressure; DBP, diastolic blood pressure; BMI, body mass index; HbAlc, glycosylated Hemoglobin; NEUT, neutrophil percentage; LYMPH, lymphocytes percentage; NC, neutrophil count; LC, lymphocyte count; RDW-CV, red cell distribution width-coefficient variation; PLT, platelet count; MPV, mean platelet volume; I-BIL, indirect bilirubin; UA, uric acid; TC, total cholesterol; TG, triglyceride; HDL-C, high-density lipoprotein cholesterol; LDL-C, low-density lipoprotein cholesterol; NLR, neutrophil-to-lymphocyte ratio; PLR, platelet-to-lymphocyte ratio.

Some clinical studies have proposed that NLR value is related to DM and its complications. Duman et al's study has demonstrated that NLR was strongly correlated with age, fasting plasma glucose and HbAlc. ${ }^{24}$ A Japanese study showed that NLR might be a potential factor for evaluating diabetic patients with a higher degree of albuminuria, ${ }^{25}$ suggesting that NLR may predict the existence of microvascular complications. ${ }^{26}$
In diabetic patients, abnormal insulin action may lead to increased platelet adhesion.

At the same time, hyperglycemia also accelerate platelet metabolism and production, exacerbating the imbalance between coagulation and anticoagulation in vivo. This process may play an important role in atherogenesis, thrombosis and microcirculation disturbance. ${ }^{27}$ PLR is reported to be a prognostic marker of inflammation for

Table 4 Binary Logistic Regression Analysis for Newly Diagnosed DPN in Follow-Up Subjects

\begin{tabular}{|l|l|l|l|l|l|l|l|}
\hline & B & S.E, & Wals & df & P & OR & 95\% Cl \\
\hline Type of diabetes & -2.398 & 1.109 & 4.677 & 1.000 & 0.031 & 0.091 & $0.010-0.799$ \\
BWI & 0.172 & 0.088 & 3.782 & 1.000 & 0.052 & 1.188 & $0.999-1.412$ \\
TC & -1.862 & 1.202 & 2.400 & 1.000 & 0.121 & 0.155 & $0.015-1.638$ \\
TG & 0.373 & 0.255 & 2.138 & 1.000 & 0.144 & 1.453 & $0.88 I-2.396$ \\
LDL-C & 2.805 & 1.537 & 3.331 & 1.000 & 0.068 & 16.526 & $0.813-336.023$ \\
NLR & -2.817 & 0.747 & 14.227 & 1.000 & 0.000 & 0.060 & $0.014-0.258$ \\
PLR & -0.011 & 0.014 & 0.669 & 1.000 & 0.413 & 0.989 & $0.962-I .016$ \\
\hline
\end{tabular}

Abbreviations: OR, odds ratio; Cl, confidence interval; BMI, body mass index; TC, total cholesterol; TG, triglyceride; LDL-C, low-density lipoprotein cholesterol; NLR, neutrophil-to-lymphocyte ratio; PLR, platelet-to-lymphocyte ratio. 
many types of cardiovascular disease, including peripheral arterial disease (PAD) and hypertension. ${ }^{28,29}$ PLR is also reported to have predictive effect about diabetes mellitus and diabetic complications in recent years. A cross-sectional study from Japan demonstrated that PLR can be a marker for high risk diabetic foot and diabetic foot ulcer in patients with type 2 diabetes. ${ }^{30}$ Besides, Duan et al's study demonstrated that the PLR was associated with proteinuria and prognosis in diabetic kidney disease (DKD) patients. ${ }^{31}$

Bilirubin is a product of heme degradation, and recent studies have reported the beneficial effects of elevated serum bilirubin on cardiovascular health and its antioxidant properties at physiological concentrations. ${ }^{32}$ Research has demonstrated that bilirubin has anti-inflammatory properties in vitro and in vivo. Bilirubin releases eNOS by inhibiting protein kinase $\mathrm{C}$ and $\mathrm{NAD}(\mathrm{P}) \mathrm{H}$ oxidase pathways that produce oxidants, and inhibits the peroxidation of lipids and lipoproteins, thereby reducing ROS and protecting nerves from damage. ${ }^{33,34} \mathrm{DPN}$ is associated with inflammatory responses, so bilirubin may have beneficial effects. Kim et $\mathrm{al}^{35}$ demonstrated a significant correlation between low serum bilirubin levels and DPN.

In our study, NLR and PLR were significantly increased in DPN in patients with type 1 diabetes. Through the ROC curve, the area under the curve of PLR was the largest. When the cut-off value was 97.880 , the sensitivity is $70.80 \%$ and the specificity was $77.30 \%$. PLR could be used to predict whether type 1 diabetes patients were associated with peripheral neuropathy. As for indirect bilirubin, this indicator is negatively correlated with DPN, which is consistent with the research results of Kim et al. According to the antioxidant and antiinflammatory properties of bilirubin, this is in line with the expected results. For patients with type 2 diabetes, NLR was significantly higher in the DPN group. According to the ROC curve, when the cut-off value is 2.485 , the sensitivity is $38.00 \%$ and the specificity was $79.00 \%$. NLR may be an independent risk factor for T2DM with DPN, as demonstrated by Siying Liu et al, $\mathrm{Xu}$ et al. ${ }^{36,37}$

Through the analysis of the results of follow-up, we found that the newly diagnosed DPN was related to the type of diabetes, BWI, inflammatory indexes, and lipid metabolism-related indexes. And the result of logistic regression analysis confirmed that the type of diabetes and NLR level were powerful indicators of risk of developing newly diagnosed DPN after adjusted other variables.
Compared with type 2 diabetes, patients with type 1 diabetes have a higher risk. While NLR value could be an effective index to predict DPN in the future.

There are some limitations in this study. For the T2DM with DPN group, it has a higher level of NLR compared with the other three groups, while the T1DM with DPN group has a higher level of PLR compared with the other three groups. The relationship between the inflammatory mechanism of diabetic peripheral neuropathy and different types of diabetes is worthy of further study. In addition, there are limitations in sample size, single center, and lack of long-term clinical observation.

Our results show that the T1DM patients who has a higher level of PLR is more likely to develop into DPN, while T2DM patients who has a higher level of NLR is more likely to develop into DPN. NLR and PLR could be used as predictors to help clinicians screening for DPN in different types of diabetes. In this study, we also found that type 1 diabetes is more likely to develop DPN in the future. For type 1 diabetes, if patients who were without DPN had higher NLR level, the risk of developing DPN in the future will be greatly increased.

\section{Data Sharing Statement}

We will share the relevant data of the paper on the website of Chinese Clinical Trial Registry within six months to one year after the paper is published.

\section{Acknowledgment}

This study was supported by the Fundamental Research Funds for the Central Universities, No. WK9110000036 (to YBW); Natural Science Foundation of Anhui Province, China, No. 1608085MH209 (to YBW); Fundamental Research Funds for the Central Universities, No. WK9110000114 (to JW).

\section{Disclosure}

The authors report no conflicts of interest in this work.

\section{References}

1. Fukuhara A, Matsuda $M$, Nishizawa $M$, et al. Visfatin: a protein secreted by visceral fat that mimics the effects of insulin. Science. 2005;307(5708):426-430. doi:10.1126/science.1097243

2. Steppan CM, Bailey ST, Bhat S, et al. The hormone resistin links obesity to diabetes. Nature. 2001;409(6818):307-312. doi:10.1038/ 35053000

3. Pop-Busui R, Boulton AJM, Feldman EL, et al. Diabetic neuropathy: a position statement by the American Diabetes Association. Diabetes Care. 2017;40(1):136-154. doi:10.2337/dc16-2042 
4. King GL. The role of inflammatory cytokines in diabetes and its complications. J Periodontol. 2008;79(8s):1527-1534. doi:10.1902/ jop.2008.080246

5. Korniluk A, Koper-Lenkiewicz OM, Kamińska J, Kemona H, Dymicka-Piekarska V. Mean Platelet Volume (MPV): new perspectives for an old marker in the course and prognosis of inflammatory conditions. Mediators Inflamm. 2019;9213074:2019.

6. Poz D, De Falco E, Pisano C, et al. Diagnostic and prognostic relevance of red blood cell distribution width for vascular aging and cardiovascular diseases. Rejuvenation Res. 2019;22(2):146-162. doi:10.1089/rej.2018.2094

7. Kim YC, Song JE, Kim EJ, et al. A simple scoring system using the red blood cell distribution width, delta neutrophil index, and platelet count to predict mortality in patients with severe sepsis and septic shock. J Intensive Care Med. 2019;34(2):133-139. doi:10.1177/ 0885066618787448

8. Montagnana M, Danese E. Red cell distribution width and cancer Ann Trans Med. 2016;4(20):399. doi:10.21037/atm.2016.10.50

9. Afari ME, Bhat T. Neutrophil to lymphocyte ratio (NLR) and cardiovascular diseases: an update. Expert Rev Cardiovasc Ther. 2016;14 (5):573-577. doi:10.1586/14779072.2016.1154788

10. Buyukkaya E, Karakaş MF, Karakaş E, et al. Correlation of neutrophil to lymphocyte ratio with the presence and severity of metabolic syndrome. Clin Appl Thromb/Hemost. 2012.

11. Diem S, Schmid S, Krapf M, et al. Neutrophil-to-Lymphocyte ratio (NLR) and Platelet-to-Lymphocyte ratio (PLR) as prognostic markers in patients with non-small cell lung cancer (NSCLC) treated with nivolumab. Lung Cancer. 2017;111:176-181. doi:10.1016/j. lungcan.2017.07.024

12. American Diabetes Association. 2. classification and diagnosis of diabetes: standards of medical care in Diabetes-2018. Diabetes Care. 2018;41(Supplement 1):S13-s27. doi:10.2337/dc18-S002

13. Tesfaye S, Boulton AJM, Dyck PJ, et al. Diabetic neuropathies: update on definitions, diagnostic criteria, estimation of severity, and treatments. Diabetes Care. 2010;33(10):2285-2293. doi:10.2337/ dc10-1303

14. Iqbal Z, Azmi S, Yadav R, et al. Diabetic peripheral neuropathy: epidemiology, diagnosis, and pharmacotherapy. Clin Ther. 2018;40 (6):828-849. doi:10.1016/j.clinthera.2018.04.001

15. Xia P, Kramer RM, King GL. Identification of the mechanism for the inhibition of $\mathrm{Na}+, \mathrm{K}(+)$-adenosine triphosphatase by hyperglycemia involving activation of protein kinase $\mathrm{C}$ and cytosolic phospholipase A2. J Clin Invest. 1995;96(2):733-740. doi:10.1172/JCI118117

16. Vareniuk I, Pavlov IA, Obrosova IG. Inducible nitric oxide synthase gene deficiency counteracts multiple manifestations of peripheral neuropathy in a streptozotocin-induced mouse model of diabetes. Diabetologia. 2008;51(11):2126-2133. doi:10.1007/s00125-008-1136-3

17. El Kebir D, Filep JG. Targeting neutrophil apoptosis for enhancing the resolution of inflammation. Cells. 2013;2(2):330-348. doi: $10.3390 /$ cells2020330

18. Rajakariar R, Lawrence T, Bystrom J, et al. Novel biphasic role for lymphocytes revealed during resolving inflammation. Blood. 2008;111(8):4184-4192. doi:10.1182/blood-2007-08-108936

19. Gibson PH, Cuthbertson BH, Croal BL, et al. Usefulness of neutrophil/lymphocyte ratio as predictor of new-onset atrial fibrillation after coronary artery bypass grafting. Am J Cardiol. 2010;105(2):186-191. doi:10.1016/j.amjcard.2009.09.007

20. Tamhane UU, Aneja S, Montgomery D, et al. Association between admission neutrophil to lymphocyte ratio and outcomes in patients with acute coronary syndrome. Am J Cardiol. 2008;102(6):653-657. doi:10.1016/j.amjcard.2008.05.006

21. Sawant AC, Adhikari P, Narra SR, et al. Neutrophil to lymphocyte ratio predicts short- and long-term mortality following revascularization therapy for ST elevation myocardial infarction. Cardiol J. 2014;21(5):500-508. doi:10.5603/CJ.a2013.0148
22. Moore MM, Chua W, Charles KA, Clarke SJ. Inflammation and cancer: causes and consequences. Clin Pharmacol Ther. 2010;87 (4):504-508. doi:10.1038/clpt.2009.254

23. Azab B, Daoud J, Naeem FB, et al. Neutrophil-to-lymphocyte ratio as a predictor of worsening renal function in diabetic patients (3-year follow-up study). Ren Fail. 2012;34(5):571-576. doi:10.3109/ 0886022X.2012.668741

24. Duman TT, Aktas G, Atak BM, et al. Neutrophil to lymphocyte ratio as an indicative of diabetic control level in type 2 diabetes mellitus. Afr Health Sci. 2019;19(1):1602-1606. doi:10.4314/ahs.v19i1.35

25. Kawamoto R, Ninomiya D, Kikuchi A, et al. Association of neutrophil-to-lymphocyte ratio with early renal dysfunction and albuminuria among diabetic patients. Int Urol Nephrol. 2019;51 (3):483-490. doi:10.1007/s11255-018-02065-2

26. Fawwad A, Butt AM, Siddiqui IA, et al. Neutrophil-to-lymphocyte ratio and microvascular complications in subjects with type 2 diabetes: pakistan's perspective. Turk J Med Sci. 2018;48:157-161. doi:10.3906/sag-1706-141

27. Jabeen F, Fawwad A, Rizvi HA, Alvi F. Role of platelet indices, glycemic control and hs-CRP in pathogenesis of vascular complications in type-2 diabetic patients. Pak J Med Sci. 2013;29(1):152-156. doi:10.12669/pjms.291.2592

28. Thomas G, Pichler M, Belaj K, et al. Platelet-to-lymphocyte ratio: a novel marker for critical limb ischemia in peripheral arterial occlusive disease patients. PLoS One. 2013;8(7):e67688. doi:10.1371/journal.pone.0067688

29. Sunbul M, Gerin F, Durmus E, et al. Neutrophil to lymphocyte and platelet to lymphocyte ratio in patients with dipper versus non-dipper hypertension. Clin Exper Hypertens. 2014;36(4):217-221. doi:10.3109/10641963.2013.804547

30. Mineoka Y, Ishii M, Hashimoto Y, et al. Platelet to lymphocyte ratio correlates with diabetic foot risk and foot ulcer in patients with type 2 diabetes. Endocr J. 2019;66(10):905-913. doi:10.1507/endocrj.EJ180477

31. Duan S, Sun L, Zhang C, et al. Association of platelet-to-lymphocyte ratio with kidney clinicopathologic features and renal outcomes in patients with diabetic kidney disease. Int Immunopharmacol. 2021;93:107413. doi:10.1016/j.intimp.2021.107413

32. Stocker R, Yamamoto Y, McDonagh AF, Glazer AN, Ames BN. Bilirubin is an antioxidant of possible physiological importance. Science. 1987;235(4792):1043-1046. doi:10.1126/science.3029864

33. Kwak JY, Takeshige K, Cheung BS, Minakami S. Bilirubin inhibits the activation of superoxide-producing NADPH oxidase in a neutrophil cell-free system. Biochim Biophys Acta. 1991;1076 (3):369-373. doi:10.1016/0167-4838(91)90478-I

34. Son SM. Reactive oxygen and nitrogen species in pathogenesis of vascular complications of diabetes. Diabetes Metab J. 2012;36 (3):190-198. doi:10.4093/dmj.2012.36.3.190

35. Kim ES, Lee SW, Mo EY, Moon SD, Han JH. Inverse association between serum total bilirubin levels and diabetic peripheral neuropathy in patients with type 2 diabetes. Endocrine. 2015;50 (2):405-412. doi:10.1007/s12020-015-0583-0

36. Liu S, Zheng H, Zhu X, et al. Neutrophil-to-lymphocyte ratio is associated with diabetic peripheral neuropathy in type 2 diabetes patients. Diabetes Res Clin Pract. 2017;130:90-97. doi:10.1016/j. diabres.2017.05.008

37. Xu T, Weng Z, Pei C, et al. The relationship between neutrophil-tolymphocyte ratio and diabetic peripheral neuropathy in Type 2 diabetes mellitus. Medicine. 2017;96(45):e8289. doi:10.1097/ MD.0000000000008289 
Journal of Pain Research

\section{Publish your work in this journal}

The Journal of Pain Research is an international, peer reviewed, open access, online journal that welcomes laboratory and clinical findings in the fields of pain research and the prevention and management of pain. Original research, reviews, symposium reports, hypothesis formation and commentaries are all considered for publication. The manuscript management system is completely online and includes a very quick and fair peer-review system, which is all easy to use. Visit http:// www.dovepress.com/testimonials.php to read real quotes from published authors.

Submit your manuscript here: https://www.dovepress.com/journal-of-pain-research-journal 